\title{
High Resolution Data Collection at S2C2, a National CryoEM Center
}

\author{
Corey Hecksel $^{1}$, Kaiming Zhang ${ }^{2}$, Grigore Pintilie ${ }^{3}$, Patrick Mitchell ${ }^{4}$, Yee-Ting Li ${ }^{1}$ and Wah Chiu ${ }^{5}$
}

${ }^{1}$ SLAC National Accelerator Laboratory, United States, ${ }^{2}$ Stanford University, United States, ${ }^{3} 1$. Department of Bioengineering, James H. Clark Center, Stanford University, Stanford, California, 94305 USA, United States, ${ }^{4}$ SLAC National Accelerator Laboratory, Stanford University, United States, ${ }^{5}$ School of Medicine, Stanford University, Stanford, California, United States

As a national facility, it is imperative that we are able to get the most out of each user's data collection session. This includes optimization of high-resolution single particle data collection throughput and quality, by optimizing data collection parameters for each user's samples. This starts with selection of the microscope used for data collection. The Stanford-SLAC cryoEM Center $\left(\mathrm{S}^{2} \mathrm{C}^{2}\right)$ has three Thermo Scientific Titan Krios G3i electron microscopes equipped with either a K3 with energy filter (Gatan, Inc), or a Falcon 4 (Thermo Scientific) direct electron detector. While users mostly have a preference for one system over the other, we have demonstrated the ability to achieve structures at a resolution of $1.34 \AA$ and $1.36 \AA$, from our Krios equipped with either an energy-filtered Gatan K3, or Thermo Scientific Falcon 4 detector without an energy filter, respectively [1]. For these structures we used $\sim 8,000$ movies $(\sim 900,000$ particles) collected over 16 hours for the K3 and 7,700 movies ( 500,000 particles) collected over 15 hours for the Falcon 4 (Figure 1A). We have also collected data with EER mode on the Falcon 4 which can extend the structure resolution to $1.27 \AA$. Figure 2 shows a 3D density map with apparent side chain features.

In addition to the selection of the detector used for single particle data collection, imaging conditions, based on the individual needs of each user's sample, are also essential to achieving the highest quality data while optimizing data collection rates. By using Aberration Free Image Shift (AFIS) to acquire multiple exposures per stage movement, we are able to collect more than 500 images an hour, yielding around 8,000 movie stacks in a 16-hour period. At $\mathrm{S}^{2} \mathrm{C}^{2}$, two of the Krios are equipped with Fringe Free Imaging (FFI) which has the potential to further increase data collection rates.

In order to monitor data collection, as well as data quality, we have implemented a bespoke data processing pipeline, that performs motion correction [2] and CTF estimation [3], outputting the results to a Slack channel so that users get real-time feedback during their data collection (Figure 1B). We are currently working to expand this to on-the-fly particle picking as well as 2D and 3D classification. This data processing pipeline allows users to monitor data in real time, making it possible to adjust data collection parameters based on real time data processing results such as particle preferred orientation, defocus distribution and data quality. It also allows staff the ability to monitor the data collection and respond to microscope errors that can occur during an automated data collection session.

Due to recent increases in data collection rates, $\mathrm{S}^{2} \mathrm{C} 2$ users routinely want to image multiple samples in a typical 24-48hr session. In order to give users more flexibility to change samples as needed, we have implemented a system for secured remote operation of our electron microscopes via the web. We are starting to roll out a program that will allow remote users to operate the microscopes for their data collection session without the need to be onsite. This allows users the ability to change samples as soon as they have collected enough data for a particular sample, the only prerequisite being that all grids must have been loaded into ThermoFisher's multigrid cassette at the start of the remote data acquisition session. 
In summary, our primary goal as a national cryoEM center is to optimize data collection for all users, so that the highest quality data can be acquired at the highest volume and throughput possible. In order to do this, we work with the user to select data collection parameters and the equipment to use, though all of these have equivalent performance capacity. In addition, we have a data processing pipeline which allows users to monitor their data collection in real time as well as tools for remote operation of the microscopes.

Acknowledgement: S2C2 is supported by the National Institutes of Health Common Fund Transformative High Resolution Cryo-Electron Microscopy program (U24 GM129541) and grant (R01GM079429).

A

\begin{tabular}{|c|c|c|}
\hline & K3 Dataset & Flacon 4 Dataset \\
\hline Microscope & Titan Krios G3i & Titan Krios G3i \\
\hline Pixel Size (Å/pixel) & 0.4 & 0.502 \\
\hline $\begin{array}{c}\text { Total Exposure } \\
\left(\mathrm{e}-/ \AA^{2}\right)\end{array}$ & 45 & 40 \\
\hline \begin{tabular}{|l|} 
Exposure Time (s) \\
\end{tabular} & 0.5 & 2 \\
\hline $\begin{array}{l}\text { Energy Filter Slit } \\
\text { Width (eV) }\end{array}$ & 15 & $\mathrm{~N} / \mathrm{A}$ \\
\hline $\begin{array}{l}\text { Defocus Range } \\
\qquad(\mu \mathrm{m})\end{array}$ & -0.35 to -1.3 & -0.35 to -1.3 \\
\hline $\begin{array}{c}\text { \# of Micrographs } \\
\text { Collected }\end{array}$ & 8,034 & 7,734 \\
\hline $\begin{array}{c}\text { \# of Micrographs } \\
\text { Used }\end{array}$ & 6,951 & 5,427 \\
\hline \# of Initial Particles & $1,176,336$ & 707,350 \\
\hline \# of Final Particles & 902,455 & 500,643 \\
\hline Resolution* (Å) & 1.34 & 1.36 \\
\hline
\end{tabular}

B

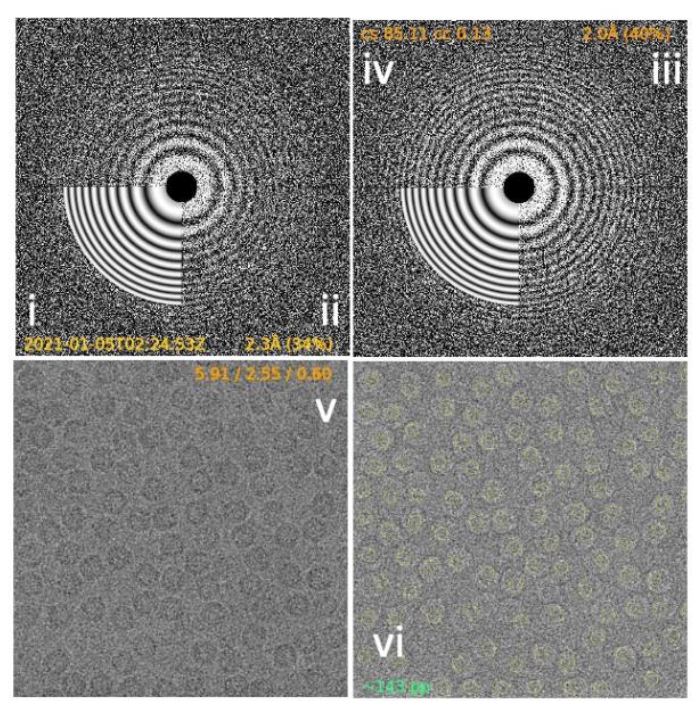

Figure 1. Apoferritin Data Collection. (A) Data collection parameters and data processing statistics for Apoferritin using the Gatan K3 and energy filter, and the Thermo Fisher Scientific Falcon 4. (B) Representative output for a single micrograph of apoferritin from our automated data processing pipeline. The top row shows a cropped FFT of the micrograph before (left) and after (right) motion correction. The bottom row shows the motion corrected micrograph (left) and the motion corrected micrograph with the contrast inverted and the positions of the picked particles shown with a yellow circle. In addition to the images, each micrograph contains the following information: (i) the data and time the image was collection, estimated resolution and percent Nyquist pre- and post-motion correction (ii and iii, respectively), (iv) astigmatism and cross correlation values, (v) RMS drift from the $1^{\text {st }} / 5^{\text {th }} /$ All frames, and (vi) the number of particles picked. 

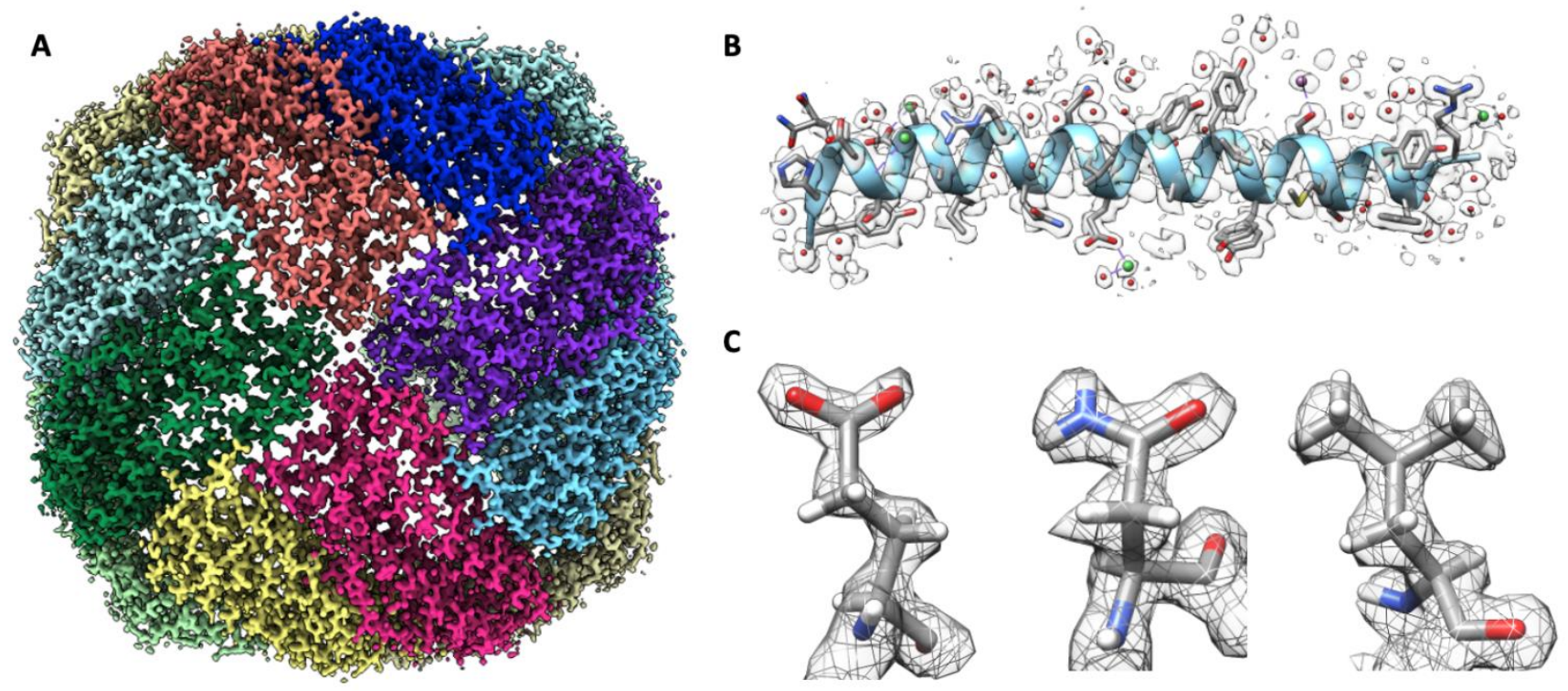

Figure 2. Apoferritin reconstruction using the Falcon 4. (A) The map of Apoferritin solved at $1.27 \AA$ resolution, showing the 24 octahedrally symmetric subunits in different colors. (B) A single helix from one of the subunits, showing resolved side chains and water. (C) Three representative side chains, hydrogen atoms can be seen in certain locations.

\section{References}

[1] Zhang, K, Pintilie, GD, Li, S, Schmid, MF, \& Chiu, W (2020) Resolving individual atoms of protein complex by cryo-electron microscopy. Cell Res 30(12):1136-1139.

[2] Shawn Q. Zheng, Eugene Palovcak, Jean-Paul Armache, Yifan Cheng and David A. Agard (2016) Anisotropic Correction of Beam-induced Motion for Improved Single-particle Electron Cryo-microscopy, Nature Methods, submitted. BioRxiv: http://biorxiv.org/content/early/2016/07/04/061960

[3] Rohou A, Grigorieff N. CTFFIND4: Fast and accurate defocus estimation from electron micrographs. J Struct Biol. 2015 Nov;192(2):216-21. doi: 10.1016/j.jsb.2015.08.008. Epub 2015 Aug 13. PMID: 26278980; PMCID: PMC6760662. 